\title{
The design of vehicle tracking based on LDC1612
}

\author{
Huang Jian \\ XiJing University, Xi'an 710123, China; \\ 565200245@qq.com
}

Keywords: Automatic tracking; LDC1612; homemade coil

Abstract: The new digital inductance sensor LDC1612 is a dual channel induction sensor. It can detect metal objects by using an inductance coil. It can detect the rotation angle and distance of metal objects. Especially suitable for automatic vehicle tracking, track by thin wire structure. In tracking, the two self inductance coil are respectively arranged on the both sides of the runway. When the wire close to a coil, eddy current based on the principle of oscillation frequency of the coil will change, and the results are converted to digital signals, transmitted to the microprocessor, through software programming, it can automatic tracking control of the car.

\section{Introduction}

Shortly after the launch of the first digital inductance sensor LDC1000 in TI company, launched the double channel digital inductance sensor LDC1612.LDC1612 with respect to LDC1000, more cost-effective, not only a channel, and the converted digital signal is as high as 28 , connected with any microprocessor through the IIC interface to facilitate the processing, and the precision is higher.

\section{Hardware Design}

\section{LDC1612 Introduction}

The pin diagram of the LDC1612 is shown in figure 1. In Figure 1, SCL and SDA are IIC interfaces, which can be easily connected with various microprocessors. The INOA and INOB are connected by an inductance coil, and the IN1A and IN1B are connected to another inductance coil. Using $3.3 \mathrm{~V}$ power supply. CLKIN is the clock input. ADDR in the application, grounding, INTB can produce interrupts. 


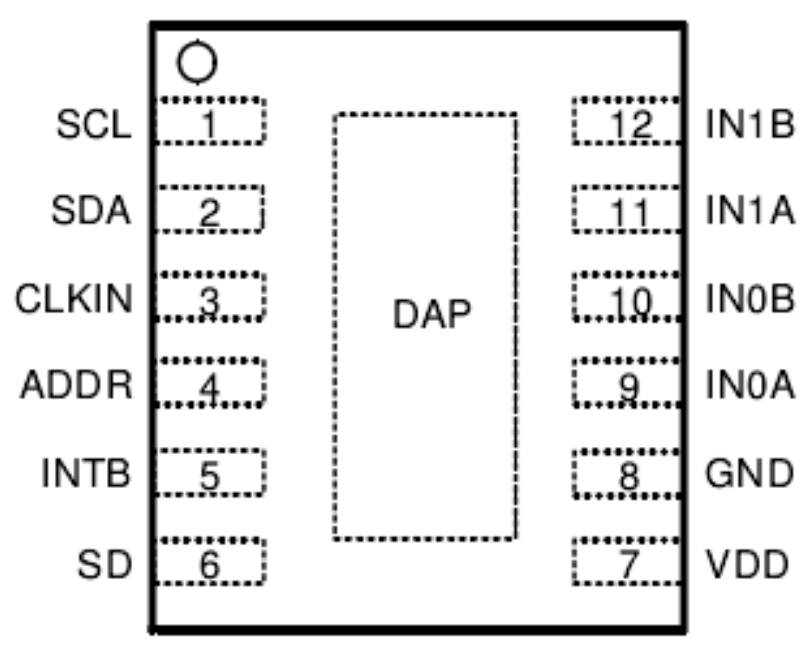

Figure 1 LDC1612 pin diagram

Figure 2 shows the connection diagram between the LDC1612 and the microprocessor. The clock input for the LDC1612 in the figure is 40MHz. INOA and INOB are connected with home-made inductors to realize detection of target metal objects. IN1A and IN1B are connected with home-made inductors to realize detection of target metal objects, and two coils realize double channel detection. The result is converted to a 28 bit digital quantity, connected to the microprocessor via SDA and SCL, and passed the results to the microprocessor.

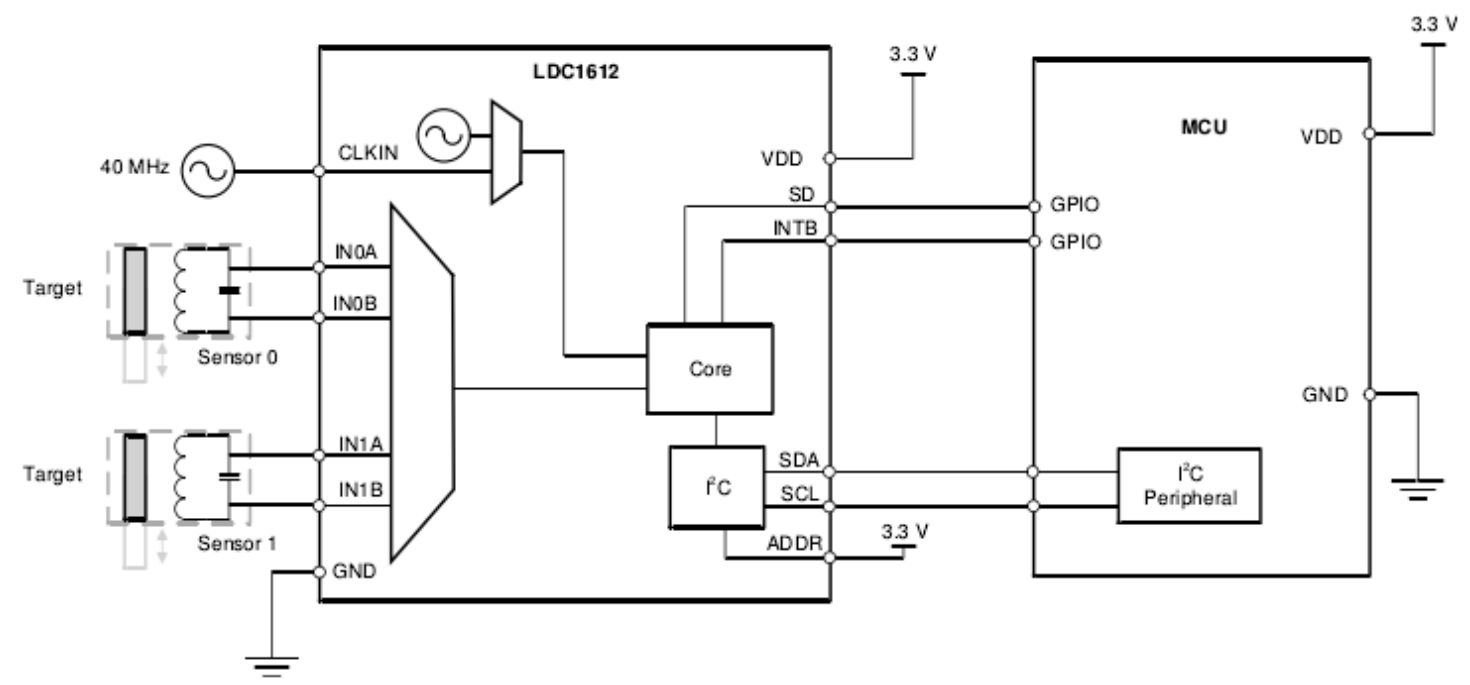

Figure 2 connection diagram of LDC1612 and microprocessor

\section{Software Program}

In this design, under KEIL 5, programming with C language. Complete the initialization of LDC1612 and the detection of metal objects, the following part of the code:

void LDC1612_Init(void) 
delay_ms(500);

LDC1612_LCD_write_16bit(LDC13xx16xx_CMD_REF_COUNT_CH0,0X04D6);

LDC1612_LCD_write_16bit(LDC13xx16xx_CMD_REF_COUNT_CH1,0X04D6);

LDC1612_LCD_write_16bit(LDC13xx16xx_CMD_REF_COUNT_CH2,0X04D6);

LDC1612_LCD_write_16bit(LDC13xx16xx_CMD_REF_COUNT_CH3,0X04D6);

LDC1612_LCD_write_16bit(LDC13xx16xx_CMD_SETTLE_COUNT_CH0,0X0A);

LDC1612_LCD_write_16bit(LDC13xx16xx_CMD_SETTLE_COUNT_CH1,0X0A);

LDC1612_LCD_write_16bit(LDC13xx16xx_CMD_SETTLE_COUNT_CH2,0X0A);

LDC1612_LCD_write_16bit(LDC13xx16xx_CMD_SETTLE_COUNT_CH3,0X0A);

LDC1612_LCD_write_16bit(LDC13xx16xx_CMD_CLOCK_DIVIDERS_CH0,0X12);

LDC1612_LCD_write_16bit(LDC13xx16xx_CMD_CLOCK_DIVIDERS_CH1,0X1002);

LDC1612_LCD_write_16bit(LDC13xx16xx_CMD_CLOCK_DIVIDERS_CH2,0X1002);

LDC1612_LCD_write_16bit(LDC13xx16xx_CMD_CLOCK_DIVIDERS_CH3,0X1002);

LDC1612_LCD_write_16bit(LDC13xx16xx_CMD_ERROR_CONFIG,0X0000);

LDC1612_LCD_write_16bit(LDC13xx16xx_CMD_DRIVE_CURRENT_CH2,0x9000);

LDC1612_LCD_write_16bit(LDC13xx16xx_CMD_DRIVE_CURRENT_CH3,0x9000);

LCD_write_16bit(LDC13xx16xx_CMD_CONFIG,0x1401);

LDC1612_LCD_write_16bit(LDC13xx16xx_CMD_CONFIG,0x1401); \}

The above code implements the initialization of LDC1612 programming.

\section{Summary}

This paper describes the design of a multi channel digital converter based on LDC1612 inductance, the ultra low power MSP430F5529 as a master, with LDC1612 as the detecting device, a careful analysis of the working principle of LDC1612 and related parameters, realize high speed data acquisition through the IIC interface, to reduce power consumption, reduce the volume, digital interface, digital high up to 28 , can realize non-contact metal detection, distance from $0 \mathrm{~mm}$ to $100 \mathrm{~mm}$, the precision is close to UM level. Because of its strong anti-interference ability, it can be used in dust, dirt, oil and humidity and other harsh environment. Its multi-channel sensing capability supports foil or conductive ink targets, providing unlimited possibilities for creative and innovative system design.

\section{Reference}

[1]Texas Instruments.LDC1612 Multi-Channel 28-Bit Inductance to Digital Converter (LDC) for Inductive Sensing[M].上海, 2014，12。 
[2]Texas Instruments.LDC Sensor Design[M].上海，2015,3

[3] Guo Yu, Li Yanmei, Wang Peng. Design of coin discrimination system of eddy current sensor based on [J] 2012,25 Journal of transducer technology, (4): 557-560

[4]TI.LDC1612 Evaluation Module User's Guide[M].AMERICAN, 2014， 17-18。

[5] Shi Yanping, Liu Chengwen, Ni Lixue. Dynamic track of amorphous alloy pressing magnetic research on the inductive stress sensor based on Journal of sensor technology

[J].2009,22 (12): 1704-1708.

[6] Bao Binghao, Jiang Feng, Zhao Zhan,, Song Xuefeng. A new weak magnetic field sensor based on the giant magneto impedance effect in amorphous band. Journal of sensing technology, [J].2006,19 (6): 2380-2383.

[7] source, money Junyue. Study on magnetic field sensor based on amorphous alloy electrical measurement and instrumentation, [J].2006,43 (2): 59-61.

[8] Fang, Dongming, Zhao Xiaolin, Wang Xining, Zhou Yong. Design and Simulation of RF solenoid micro inductors, Journal of sensing technology

[J].2006,19 (5): 1878-1880.

[9], Shen Jianhua, Yang Yanqin, et al..MSP430 series 16 bit ultra-low power MCU principle and application [M]. Beijing: Tsinghua University press, 2009:48-52

[10] Wang Peng, Qin Daoyu, Ding Tianhuai, Fu Zhibin. Research on signal detection technique of micro planar coil inductor, Journal of transducer technology

[J]., 2007,20 (10): 2333-2336. 